\title{
A NONTRIVIAL ALGEBRAIC CYCLE IN THE JACOBIAN VARIETY OF THE KLEIN QUARTIC
}

\author{
YUUKI TADOKORO
}

\begin{abstract}
We prove some value of the harmonic volume for the Klein quartic $C$ is nonzero modulo $\frac{1}{2} \mathbb{Z}$, using special values of the generalized hypergeometric function ${ }_{3} F_{2}$. This result tells us the algebraic cycle $C-C^{-}$is not algebraically equivalent to zero in the Jacobian variety $J(C)$.
\end{abstract}

\section{INTRODUCTION}

Let $X$ be a compact Riemann surface of genus $g \geq 2$ and $J(X)$ its Jacobian variety. By the Abel-Jacobi map $X \rightarrow J(X), X$ is embedded in $J(X)$. The algebraic 1-cycle $X-X^{-}$in $J(X)$ is homologous to zero. Here we denote by $X^{-}$the image of $X$ under the multiplication map by -1 . If $X$ is hyperelliptic, $X=X^{-}$in $J(X)$. For the rest of this paper, suppose $g \geq 3$. B. Harris [5] studied the problem whether the cycle $X-X^{-}$in $J(X)$ is algebraically equivalent to zero or not. The harmonic volume $I$ for $X$ was introduced by Harris 4, using Chen's iterated integrals 2. Let $H$ denote the first integral homology group $H_{1}(X ; \mathbb{Z})$ of $X$. The harmonic volume $I$ is defined to be a homomorphism $\left(H^{\otimes 3}\right)^{\prime} \rightarrow \mathbb{R} / \mathbb{Z}$. Here $\left(H^{\otimes 3}\right)^{\prime}$ is a certain subgroup of $H^{\otimes 3}$. See Section 2 for the definition. Let $\omega$ be a third tensor product of holomorphic 1-forms on $X$. Suppose that $\omega+\bar{\omega}$ and $(\omega-\bar{\omega}) / \sqrt{-1}$ belong to $\left(H^{\otimes 3}\right)^{\prime}$. If the cycle $X-X^{-}$is algebraically equivalent to zero, then twice the values at both $\omega+\bar{\omega}$ and $(\omega-\bar{\omega}) / \sqrt{-1}$ of the harmonic volume are zero modulo $\mathbb{Z}$. Harris proved twice the value at $\omega+\bar{\omega}$ of the harmonic volume for the Fermat quartic $F(4)$ are nonzero modulo $\mathbb{Z}$. This implies $F(4)-F(4)^{-}$is not algebraically equivalent to zero in $J(F(4))([5,6])$. Ceresa [1] showed that $X-X^{-}$is not algebraically equivalent to zero for a generic $X$. We know few explicit nontrivial examples except for $F(4)$. Let $C$ denote the Klein quartic. See Section 4.1 for the definition. The aim of this paper is to show

Theorem 4.14. The algebraic cycle $C-C^{-}$is not algebraically equivalent to zero in the Jacobian variety $J(C)$.

Since Harris used the special feature of $F(4)$ that its normalized period matrix has entries in $\mathbb{Z}[\sqrt{-1}]$, it is not difficult to find some $\omega$ so that $\omega+\bar{\omega}$ and $(\omega-\bar{\omega}) / \sqrt{-1}$ belong to $\left(H^{\otimes 3}\right)^{\prime}$ for $F(4)$. But, in general, it is not easy to find such an $\omega$. For the Klein quartic $C$, we prove $(D+\bar{D}) / 7$ and $(D-\bar{D}) / \sqrt{-7}$ belong to $\left(H^{\otimes 3}\right)^{\prime}$ (Proposition 4.7). See Section 4.3 for the definitions of them. In Theorem 4.9 we compute the value at $(D-\bar{D}) / \sqrt{-7} \in\left(H^{\otimes 3}\right)^{\prime}$ of the harmonic volume for $C$

$$
I((D-\bar{D}) / \sqrt{-7})=\frac{28}{\sqrt{-7}}\left(\frac{\zeta_{7}^{2}-\zeta_{7}^{6}}{\zeta_{7}+1} x_{1,2}+\frac{\zeta_{7}^{4}-\zeta_{7}^{5}}{\zeta_{7}^{2}+1} x_{2,3}+\frac{\zeta_{7}-\zeta_{7}^{3}}{\zeta_{7}^{4}+1} x_{3,1}\right) \bmod \mathbb{Z} .
$$

Here, $\zeta_{7}=\exp (2 \pi \sqrt{-1} / 7)$ and $x_{i, j}$ 's are real constants obtained from some special values of the generalized hypergeometric function ${ }_{3} F_{2}$ (Lemma 4.13). By numerical computation using MATHEMATICA, we obtain Theorem 4.14 We give a calculation program in Appendix.

2000 Mathematics Subject Classification. 14H30, 14H40, 30F30, 32G15. 
Acknowledgments. The author is grateful to Nariya Kawazumi for valuable advice and reading the manuscript. Masahiko Yoshinaga and Shuji Yamamoto suggest useful ideas for the proof of Proposition 4.7 to him. He would like to thank Masaaki Suzuki for his helpful comments for MATHEMATICA programs. This work is partially supported by 21st Century COE program (University of Tokyo) by the Ministry of Education, Culture, Sports, Science and Technology.

\section{The HARMONiC VOlume}

We recall the harmonic volume for a compact Riemann surface $X$ of genus $g \geq 3$ 4. We identify the first integral homology group $H_{1}(X ; \mathbb{Z})$ of $X$ with the first integral cohomology group by Poincaré duality, and denote it by $H$. Moreover we identify $H$ with the space of all the real harmonic 1-forms on $X$ with integral periods. Let $K$ be the kernel of the intersection pairing $():, H \otimes_{\mathbb{Z}} H \rightarrow \mathbb{Z}$. For the rest of this paper, we write $\otimes=\otimes_{\mathbb{Z}}$, unless otherwise stated. The Hodge star operator $*$ on the space of all the 1-forms $A^{1}(X)$ is locally given by $*\left(f_{1}(z) d z+f_{2}(z) d \bar{z}\right)=-\sqrt{-1} f_{1}(z) d z+\sqrt{-1} f_{2}(z) d \bar{z}$ in a local coordinate $z$ and depends only on the complex structure and not on the choice of Hermitian metric. For any $\sum_{i=1}^{n} a_{i} \otimes b_{i} \in K$, there exists a unique $\eta \in A^{1}(X)$ such that $d \eta=\sum_{i=1}^{n} a_{i} \wedge b_{i}$ and $\int_{X} \eta \wedge * \alpha=0$ for any closed 1 -form $\alpha \in A^{1}(X)$. Here $a_{i}$ and $b_{i}$ are regarded as real harmonic 1 -forms on $X$. Choose a point $x_{0} \in X$.

Definition 2.1. (The pointed harmonic volume 9])

For $\sum_{i=1}^{n} a_{i} \otimes b_{i} \in K$ and $c \in H$, the pointed harmonic volume defined to be

$$
I_{x_{0}}\left(\left(\sum_{i=1}^{n} a_{i} \otimes b_{i}\right) \otimes c\right)=\sum_{i=1}^{n} \int_{\gamma} a_{i} b_{i}-\int_{\gamma} \eta \bmod \mathbb{Z} .
$$

Here $\eta \in A^{1}(X)$ is associated to $\sum_{i=1}^{n} a_{i} \otimes b_{i}$ in the way stated above and $\gamma$ is a loop in $X$ with the base point $x_{0}$ whose homology class is equal to $c$. The integral $\int_{\gamma} a_{i} b_{i}$ is Chen's iterated integral [2], that is, $\int_{\gamma} a_{i} b_{i}=\int_{0 \leq t_{1} \leq t_{2} \leq 1} f_{i}\left(t_{1}\right) g_{i}\left(t_{2}\right) d t_{1} d t_{2}$ for $\gamma^{*} a_{i}=f_{i}(t) d t$ and $\gamma^{*} b_{i}=g_{i}(t) d t$. Here $t$ is the coordinate in the interval $[0,1]$.

The harmonic volume is given as a restriction of the pointed harmonic volume $I_{x_{0}}$. We denote by $\left(H^{\otimes 3}\right)^{\prime}$ the kernel of a natural homomorphism $p: H^{\otimes 3} \rightarrow H^{\oplus 3}$ defined by $p(a \otimes b \otimes c)=$ $((a, b) c,(b, c) a,(c, a) b)$. The harmonic volume $I$ for $X$ is a linear form on $\left(H^{\otimes 3}\right)^{\prime}$ with values in $\mathbb{R} / \mathbb{Z}$ defined by the restriction of $I_{x_{0}}$ to $\left(H^{\otimes 3}\right)^{\prime}$, i.e., $I=\left.I_{x_{0}}\right|_{\left(H^{\otimes 3}\right)^{\prime}}$. Harris 4 proved that the harmonic volume $I$ is independent of the choice of the base point $x_{0}$. We have $I\left(\sum_{i} h_{\sigma(1), i} \otimes h_{\sigma(2), i} \otimes h_{\sigma(3), i}\right)=\operatorname{sgn}(\sigma) I\left(\sum_{i} h_{1, i} \otimes h_{2, i} \otimes h_{3, i}\right) \bmod \mathbb{Z}$, where $\sum_{i} h_{1, i} \otimes h_{2, i} \otimes h_{3, i} \in$ $\left(H^{\otimes 3}\right)^{\prime}$ and $\sigma$ is an element of the third symmetric group $S_{3}$. See Harris 4] and Pulte [9] for details.

In general, it is difficult to compute the correction term $\eta$ in Definition 2.1 If $X$ is a hyperelliptic curve, we have an explicit formula for the 1-form $\eta$ given by Harris [4]. This allows us to calculate the harmonic volumes for all the hyperelliptic curves (Tadokoro [11]). In this paper, we deal with the case $\eta$ vanishes.

\section{The algebraic Cycle $X-X^{-}$And an intermediate Jacobian}

We review a relation between the algebraic cycle $X-X^{-}$and the harmonic volume $I$. 
Let $j_{2}: H^{\otimes 3} \rightarrow \wedge^{3} H$ be a natural homomorphism $j_{2}(a \otimes b \otimes c)=a \wedge b \wedge c$, where $\wedge^{3} H$ denotes the third exterior power of $H$. We have the homomorphism of short exact sequences

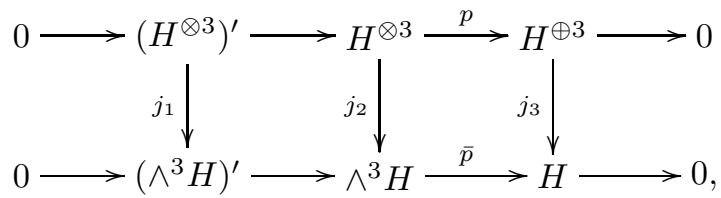

where $j_{3}(a, b, c)=a+b+c, \bar{p}(a \wedge b \wedge c)=(a, b) c+(b, c) a+(c, a) b$ and $j_{1}$ is the restriction homomorphism of $j_{2}$ to $\left(H^{\otimes 3}\right)^{\prime}$. Let $\mathcal{A}_{0}^{k}(J)$ be the space of algebraic $k$-cycles homologous to zero on the Jacobian variety $J=J(X)$, modulo rational equivalence. The Abel-Jacobi map of Griffiths $\Phi_{\mathbb{R}}: \mathcal{A}_{0}^{k}(J) \rightarrow \operatorname{Hom}_{\mathbb{Z}}\left(H^{2 k+1}(J ; \mathbb{Z}), \mathbb{R} / \mathbb{Z}\right)$ is defined by

$$
\partial W \mapsto\left\{\omega \mapsto \int_{W} \omega\right\}
$$

where $\omega$ is a harmonic $(2 k+1)$-form on $J$ with integral periods (Section 4 in 9 ). Here, the module $\operatorname{Hom}_{\mathbb{Z}}\left(H^{2 k+1}(J ; \mathbb{Z}), \mathbb{R} / \mathbb{Z}\right)$ can be identified with an intermediate Jacobian of $H_{2 k+1}(J ; \mathbb{Z})$ 9. From now on, we consider the case $k=1$. Let $\nu$ denote the Abel-Jacobi image $\Phi_{\mathbb{R}}\left(X-X^{-}\right)$. Harris (Proposition 2.1 in [6] , 4]) proved that $\left(\wedge^{3} H\right)^{\prime}$ can be identified with the primitive subgroup of $H^{3}(J ; \mathbb{Z})$ in the sence of Lefchetz, denoted by $H_{\text {prim }}^{3}(J ; \mathbb{Z})$. Using this identification and the natural projection $\operatorname{Hom}_{\mathbb{Z}}\left(H^{3}(J ; \mathbb{Z}), \mathbb{R} / \mathbb{Z}\right) \rightarrow \operatorname{Hom}_{\mathbb{Z}}\left(H_{\text {prim }}^{3}(J ; \mathbb{Z}), \mathbb{R} / \mathbb{Z}\right)$, we consider $\nu$ as an element of $\operatorname{Hom}_{\mathbb{Z}}\left(\left(\wedge^{3} H\right)^{\prime}, \mathbb{R} / \mathbb{Z}\right)$ (Section 4 and 6 in $[9]$ ).

Theorem 3.1. (Harris [4, 6]). The Abel-Jacobi image $\nu$ satisfies the commutative diagram

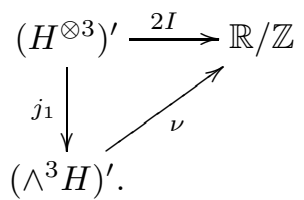

We say the algebraic cycle $X-X^{-}$is algebraically equivalent to zero in $J$ if there exists a topological 3-chain $W$ such that $\partial W=X-X^{-}$and $W$ lies on $S$, where $S$ is an algebraic (or complex analytic) subset of $J$ of complex dimension 2 (Harris [6]). The chain $W$ is unique up to 3 -cycles. We denote by $H^{1,0}$ the space of all the holomorphc 1 -forms on $X$. From [5], 2.6 in [6] and 533-534 in [13, we have

Proposition 3.2. Let $\omega \in\left(H^{1,0}\right)^{\otimes_{\mathbb{C}} 3}$ satisfying that $\omega+\bar{\omega}$ and $(\omega-\bar{\omega}) / \sqrt{-1} \in\left(H^{\otimes 3}\right)^{\prime}$. If $X-X^{-}$is algebraically equivalent to zero in $J$, then twice the values at both $\omega+\bar{\omega}$ and $(\omega-\bar{\omega}) / \sqrt{-1}$ of the harmonic volume are zero modulo $\mathbb{Z}$.

Proof. Since $X-X^{-}$is algebraically equivalent to zero in $J$, there exist a 3 -chain $W$ and an algebraic subset $S$ satisfying the above conditions. Let $H_{\mathbb{C}}$ denote $H \otimes \mathbb{C}$. Theorem 3.1 gives

$$
\left.2 I(\omega+\bar{\omega})=\int_{W} j_{1}(\omega+\bar{\omega}) \text { and } 2 I((\omega-\bar{\omega}) / \sqrt{-1})\right)=\int_{W} j_{1}(\omega-\bar{\omega}) / \sqrt{-1} .
$$

It is clear that $j_{1}(\omega)$ and $j_{1}(\bar{\omega})$ are $(3,0)$ and $(0,3)$-form in $H^{3}(J ; \mathbb{C})=\wedge^{3} H_{\mathbb{C}}$ respectively. Since $\operatorname{dim}_{\mathbb{C}} S=2$, the restriction of them to $S$ are clearly zero.

If twice the value at $\omega+\bar{\omega}$ or $(\omega-\bar{\omega}) / \sqrt{-1}$ of the harmonic volume is nonzero modulo $\mathbb{Z}$, then $X-X^{-}$is not algebraically equivalent to zero in $J$. See Hain [3], Pirola [10 and their references for the algebraic cycle $X-X^{-}$in $J$. 


\section{Some values of the harmonic volume for the Klein Quartic}

We compute some values of the harmonic volume for the Klein quartic to prove the main theorem (Theorem 4.14).

4.1. A 1-dimensional homology basis of the Klein quartic. We denote by $C$ the Klein quartic which is, by definition, the plane curve $C:=\left\{(X: Y: Z) \in \mathbb{C} P^{2} ; X^{3} Y+Y^{3} Z+Z^{3} X=\right.$ $0\}$. It is a compact Riemann surface of genus 3. It is known that the holomorphic automorphism group of $C$, Aut $(C)$, is isomorphic to $\mathrm{PSL}_{2}\left(\mathbb{F}_{7}\right)$. See 7$]$ for the details of the Klein quartic. Let $x$ and $y$ denote $X^{3} Y^{-2} Z^{-1}+1$ and $-X Y^{-1}$ respectively. The equation $X^{3} Y+Y^{3} Z+Z Z^{3} X=0$ induces $y^{7}=x(1-x)^{2}$. The holomorphic map $\pi: C \rightarrow \mathbb{C} P^{1}$ is defined by $\pi(x, y)=x$, which is a 7 -sheeted covering $C \rightarrow \mathbb{C} P^{1}$, branched over 3 branch points $\{0,1, \infty\}$. Let $\zeta_{7}$ denote $\exp (2 \pi \sqrt{-1} / 7)$. For $t \in[0,1]$, we define a loop $e_{0}:[0,1] \rightarrow C$ by $e_{0}(t)=\left(t, y_{0}(t)\right)$, where $y_{0}(t)$ is a real analytic function $\sqrt[7]{t(1-t)^{2}}$. Let $\sigma: C \rightarrow C$ be a holomorphic automorphism $\sigma(x, y)=\left(x, \zeta_{7} y\right)$. For $k=0,1, \ldots, 6$, we define loops in $C$ by $c_{k}=\sigma_{*}^{k}\left(e_{0}\right) \cdot e_{0}^{-1}$. We denote $\ell_{k}=\sigma_{*}^{k-1}\left(e_{0}\right) \cdot \sigma_{*}^{k}\left(e_{0}\right)^{-1}, k=0,1, \ldots, 7$. The loop $\ell_{0}$ can be identified with $\ell_{7}$. By abuse of notation, the homology classes of $c_{k}$ and $\ell_{k}$ are denoted by $c_{k}$ and $\ell_{k} \in H_{1}(C ; \mathbb{Z})$ respectively. Let $():, H_{1}(C ; \mathbb{Z}) \otimes H_{1}(C ; \mathbb{Z}) \rightarrow \mathbb{Z}$ be the intersection pairing, i.e., a non-degenerate bilinear form on $H_{1}(C ; \mathbb{Z})$. Tretkoff and Tretkoff 12 proved

$$
\left(c_{1}, c_{k}\right)= \begin{cases}0 & \text { if } \quad k=1,2,4,6 \\ 1 & \text { if } \quad k=3,5\end{cases}
$$

using the Hurwitz system of the branched covering $\pi$. By the definition of $\ell_{k}$, we have

$$
\left(\ell_{1}, \ell_{k}\right)=\left(c_{1}, c_{k}\right)-\left(c_{1}, c_{k-1}\right)=\left\{\begin{array}{ccc}
0 & \text { if } & k=1,2 \\
1 & \text { if } & k=3,5 \\
-1 & \text { if } & k=4,6
\end{array}\right.
$$

Moreover, we obtain that $\sigma_{*}\left(\ell_{k}\right)=\ell_{k+1}$ and $\left(\ell_{i}, \ell_{j}\right)=\left(\sigma_{*}\left(\ell_{i}\right), \sigma_{*}\left(\ell_{j}\right)\right)=\left(\ell_{i+1}, \ell_{j+1}\right)$. The intersection matrix $K^{\prime}$ of $\ell_{k}, k=1,2, \ldots, 6$ is given by

$$
\left(\begin{array}{cccccc}
0 & 0 & 1 & -1 & 1 & -1 \\
0 & 0 & 0 & 1 & -1 & 1 \\
-1 & 0 & 0 & 0 & 1 & -1 \\
1 & -1 & 0 & 0 & 0 & 1 \\
-1 & 1 & -1 & 0 & 0 & 0 \\
1 & -1 & 1 & -1 & 0 & 0
\end{array}\right)
$$

i.e., its $(i, j)$-th entry is $\left(\ell_{i}, \ell_{j}\right)$. It is easy to prove $\operatorname{det} K^{\prime}=1$ and $\left\{\ell_{k}\right\}_{k=1,2, \ldots, 6} \subset H_{1}(C$; $\mathbb{Z})$ is a basis of $H_{1}(C ; \mathbb{Z})$.

4.2. Poincaré dual of the Klein quartic. Let $\omega_{1}^{\prime}, \omega_{2}^{\prime}$ and $\omega_{3}^{\prime}$ be holomorphic 1-forms on $C,(1-x) d x / y^{6},(1-x) d x / y^{5}$ and $d x / y^{3}$ respectively. It is known that $\left\{\omega_{i}^{\prime}\right\}_{i=1,2,3}$ is a basis of the space of all the holomorphic 1-forms on $C$. The beta function $B(u, v)$ is defined by $\int_{0}^{1} t^{u-1}(1-t)^{v-1} d t$ for $u, v>0$. We denote $\left(h_{1}, h_{2}, h_{3}, h_{4}\right)=(1 / 7,2 / 7,4 / 7,1 / 7)$ and $\xi_{i}=\zeta_{7}^{7 h_{i}}$. From the equations $\sigma^{*} \omega_{i}^{\prime}=\xi_{i} \omega_{i}$ and $\int_{e_{0}} \omega_{i}^{\prime}=B\left(h_{i}, h_{i+1}\right)$, we have

Lemma 4.1.

$$
\int_{\ell_{k}} \omega_{i}^{\prime}=\left(\xi_{i}^{k-1}-\xi_{i}^{k}\right) B\left(h_{i}, h_{i+1}\right)
$$

Remark 4.2. These integrals depend only on the cohomology class of $\omega_{j}^{\prime}$ and the homology class of $\ell_{k}$. 
We set $B_{i}^{\prime}=B\left(h_{i}, h_{i+1}\right)$ and $\omega_{i}=\omega_{i}^{\prime} / B_{i}^{\prime}, i=1,2,3$. We write $L_{k}:=\sum_{i=1}^{7} \zeta_{7}^{i k} \ell_{k} \in H_{1}(C ; \mathbb{C})$ and denote the Poincaré dual by P.D.: $H^{1}(C ; \mathbb{C}) \rightarrow H_{1}(C ; \mathbb{C})$.

Proposition 4.3. We denote $\lambda_{i}=-1 /\left(\xi_{i}^{3}\left(\xi_{i}^{2}+1\right)\right) \in \mathbb{C}$. Then, we have

$$
\text { P.D. }\left(\omega_{i}\right)=\lambda_{i} L_{7 h_{i}} \text {. }
$$

Proof. Since $\sigma_{*}\left(\ell_{k}\right)=\ell_{k+1}$, we obtain $\sigma_{*} L_{k}=\zeta_{7}^{-k} L_{k}$. The eigenvalues and eigenvectors of the action of $\sigma$ on the $\mathbb{C}$-vector space $H_{1}(C ; \mathbb{C})$ are $\zeta_{7}^{-k}$ and $L_{k}$ for $k=1,2, \ldots, 6$. We have

$$
\sigma_{*}\left(\text { P.D. }\left(\omega_{i}\right)\right)=\text { P.D. }\left(\left(\sigma^{-1}\right)^{*} \omega_{i}\right)=\xi_{i}^{-1} \text { P.D. }\left(\omega_{i}\right)=\zeta_{7}^{-7 h_{i}} \text { P.D. }\left(\omega_{i}\right) .
$$

There exists a constant $\lambda_{i} \in \mathbb{C}$ such that P.D. $\left(\omega_{i}\right)=\lambda_{i} L_{7 h_{i}}$. The result follows from Lemma 4.1 and the equation

$$
\int_{\ell_{1}} \omega_{i}=\left(\text { P.D. }\left(\omega_{i}\right), \ell_{1}\right)=\left(\lambda_{i} L_{7 h_{i}}, \ell_{1}\right)=\lambda_{i}\left(L_{7 h_{i}}, \ell_{1}\right)=-\lambda_{i}\left(1-\xi_{i}\right)\left(\xi_{i}^{3}\left(\xi_{i}^{2}+1\right)\right) .
$$

Remark 4.4. We have P.D. $\left(\bar{\omega}_{i}\right)=\bar{\lambda}_{i} \bar{L}_{7 h_{i}}$. It immediately follows $\lambda_{1} \lambda_{2} \lambda_{3}=-1$.

4.3. Some values of the harmonic volume for the Klein quartic. For $t \in[0,1]$, let $f_{i}$ be a real 1 -form on $[0,1]$ defined by $e_{0}^{*} \omega_{i}^{\prime}=t^{h_{i}-1}(1-t)^{h_{i+1}-1} d t, i=1,2,3$. Let $x_{i, j}$ denote an iterated integral $\int_{e_{0}} \omega_{i} \omega_{j}=\int_{\gamma} f_{i} f_{j} /\left(B_{i}^{\prime} B_{j}^{\prime}\right)$. Here, $\gamma$ is the path $[0,1] \ni t \mapsto t \in[0,1]$. We compute the iterated integrals of $\omega_{1}, \omega_{2}$ and $\omega_{3}$ along the loop $\ell_{k}$.

Lemma 4.5. We consider $\ell_{k}$ as loops with the base point $(x, y)=(0,0) \in C$. We have

$$
\int_{\ell_{k}} \omega_{i} \omega_{j}=\left(\xi_{i} \xi_{j}\right)^{k-1}\left(1-\xi_{i} \xi_{j}\right) x_{i, j}+\left(\xi_{i} \xi_{j}\right)^{k-1}\left(\xi_{i} \xi_{j}-\xi_{j}\right) .
$$

Remark 4.6. Since $\omega_{i}$ is closed and $\omega_{i} \wedge \omega_{j}=0$, these iterated integrals are invariant under homotopy with fixed endpoints.

Proof. Using the shuffle product formula (Chen [2, 1.6) and the equations

$$
0=\int_{e_{0} \cdot e_{0}^{-1}} \omega_{i} \omega_{j}=\int_{e_{0}} \omega_{i} \omega_{j}+\int_{e_{0}^{-1}} \omega_{i} \omega_{j}+\int_{e_{0}} \omega_{i} \int_{e_{0}^{-1}} \omega_{j} \quad \text { and } \quad \int_{e_{0}} \omega_{i}=\int_{e_{0}} \omega_{i}^{\prime} / B_{i}^{\prime}=1,
$$

we have

$$
\begin{aligned}
\int_{\ell_{k}} \omega_{i} \omega_{j} & =\int_{\sigma_{*}^{k-1}\left(e_{0}\right) \cdot \sigma_{*}^{k}\left(e_{0}\right)^{-1}} \omega_{i} \omega_{j} \\
& =\int_{\sigma_{*}^{k-1}\left(e_{0}\right)} \omega_{i} \omega_{j}+\int_{\sigma_{*}^{k}\left(e_{0}\right)^{-1}} \omega_{i} \omega_{j}+\int_{\sigma_{*}^{k-1}\left(e_{0}\right)} \omega_{i} \int_{\sigma_{*}^{k}\left(e_{0}\right)^{-1}} \omega_{j} \\
& =\left(\xi_{i} \xi_{j}\right)^{k-1} \int_{e_{0}} \omega_{i} \omega_{j}+\left(\xi_{i} \xi_{j}\right)^{k} \int_{e_{0}^{-1}} \omega_{i} \omega_{j}-\xi_{i}^{k-1} \xi_{j}^{k} \int_{e_{0}} \omega_{i} \int_{e_{0}} \omega_{j} \\
& =\left(\xi_{i} \xi_{j}\right)^{k-1} \int_{e_{0}} \omega_{i} \omega_{j}+\left(\xi_{i} \xi_{j}\right)^{k}\left\{-\int_{e_{0}} \omega_{i} \omega_{j}+\int_{e_{0}} \omega_{i} \int_{e_{0}} \omega_{j}\right\}-\xi_{i}^{k-1} \xi_{j}^{k} \\
& =\left(\xi_{i} \xi_{j}\right)^{k-1}\left(1-\xi_{i} \xi_{j}\right) \int_{e_{0}} \omega_{i} \omega_{j}+\left(\xi_{i} \xi_{j}\right)^{k-1}\left(\xi_{i} \xi_{j}-\xi_{j}\right) .
\end{aligned}
$$

The subset $\mathcal{H}$ of $\left(H^{\otimes 3}\right)^{\prime} \otimes \mathbb{R}$ is defined by $\left\{\omega+\bar{\omega},(\omega-\bar{\omega}) / \sqrt{-1} ; \omega \in H^{1,0} \otimes_{\mathbb{C}} H^{1,0} \otimes_{\mathbb{C}} H^{1,0}\right\}$. We will find some elements of $\mathcal{H} \cap\left(H^{\otimes 3}\right)^{\prime}$. Let $D$ and $\bar{D}$ denote $\sum_{\mu \in S_{3}} \operatorname{sgn}(\mu) \omega_{\mu(1)} \otimes_{\mathbb{C}} \omega_{\mu(2)} \otimes_{\mathbb{C}} \omega_{\mu(3)}$ and $\sum_{\mu \in S_{3}} \operatorname{sgn}(\mu) \bar{\omega}_{\mu(1)} \otimes_{\mathbb{C}} \bar{\omega}_{\mu(2)} \otimes_{\mathbb{C}} \bar{\omega}_{\mu(3)} \in\left(H_{\mathbb{C}}\right)^{\otimes_{\mathbb{C}} 3}$ respectively. Using Proposition 4.3 and 
Remark 4.4] $D$ and $\bar{D}$ are identified with $-\sum_{\mu \in S_{3}} \operatorname{sgn}(\mu) L_{7 h_{\mu(1)}} \otimes_{\mathbb{C}} L_{7 h_{\mu(2)}} \otimes_{\mathbb{C}} L_{7 h_{\mu(3)}}$ and $-\sum_{\mu \in S_{3}} \operatorname{sgn}(\mu) \bar{L}_{7 h_{\mu(1)}} \otimes_{\mathbb{C}} \bar{L}_{7 h_{\mu(2)}} \otimes_{\mathbb{C}} \bar{L}_{7 h_{\mu(3)}}$ respectively. The coefficients of $\ell_{p} \otimes_{\mathbb{C}} \ell_{q} \otimes_{\mathbb{C}} \ell_{r}$ of $D$ and $\bar{D}$ are

$$
\alpha_{p, q, r}=-\left|\begin{array}{lll}
\zeta_{7}^{p} & \zeta_{7}^{2 p} & \zeta_{7}^{4 p} \\
\zeta_{7}^{q} & \zeta_{7}^{2 q} & \zeta_{7}^{4 q} \\
\zeta_{7}^{r} & \zeta_{7}^{2 r} & \zeta_{7}^{4 r}
\end{array}\right| \text { and } \bar{\alpha}_{p, q, r}=-\left|\begin{array}{lll}
\zeta_{7}^{6 p} & \zeta_{7}^{5 p} & \zeta_{7}^{3 p} \\
\zeta_{7}^{6 q} & \zeta_{7}^{5 q} & \zeta_{7}^{3 q} \\
\zeta_{7}^{6 r} & \zeta_{7}^{5 r} & \zeta_{7}^{3 r}
\end{array}\right|
$$

respectively. It is trivial that $D+\bar{D}$ and $(D-\bar{D}) / \sqrt{-1} \in \mathcal{H}$. Furthermore, we have

Proposition 4.7. $(D+\bar{D}) / 7$ and $(D-\bar{D}) / \sqrt{-7} \in\left(H^{\otimes 3}\right)^{\prime}$.

Proof. It suffices to prove that $\alpha_{p, q, r}$ belongs to the principal ideal $(\sqrt{-7}) \mathbb{Z}[(1+\sqrt{-7}) / 2] \subset$ $\mathbb{Z}[(1+\sqrt{-7}) / 2]$. It is well known that $\operatorname{Gal}\left(\mathbb{Q}\left(\zeta_{7}\right) / \mathbb{Q}\right) \cong\left\{\sigma_{i}\right\}_{i=1,2, \ldots, 6} \cong \mathbb{Z} / 6 \mathbb{Z}$, where $\sigma_{i}\left(\zeta_{7}\right)=\zeta_{7}^{i}$. Since $[\mathbb{Q}(\sqrt{-7}): \mathbb{Q}]=2$, we obtain $\operatorname{Gal}\left(\mathbb{Q}\left(\zeta_{7}\right) / \mathbb{Q}(\sqrt{-7})\right)$, the subgroup of $\operatorname{Gal}\left(\mathbb{Q}\left(\zeta_{7}\right) / \mathbb{Q}\right)$, is generated by $\sigma_{2}$. It is clear that $\alpha_{p, q, r}$ is invariant under the action of $\sigma_{2}$. So, we have $\alpha_{p, q, r} \in \mathbb{Q}(\sqrt{-7})$. On the other hand, it immediately follows $\alpha_{p, q, r}$ belongs to the principal ideal $\left(\zeta_{7}-1\right) \mathbb{Z}\left[\zeta_{7}\right] \subset \mathbb{Z}\left[\zeta_{7}\right]$. Therefore, we have

$$
\alpha_{p, q, r} \in \mathbb{Q}(\sqrt{-7}) \cap\left(\zeta_{7}-1\right) \mathbb{Z}\left[\zeta_{7}\right]=(\sqrt{-7}) \mathbb{Z}[(1+\sqrt{-7}) / 2] \subset \mathbb{Z}[(1+\sqrt{-7}) / 2] .
$$

We have $\alpha_{p, q, r}+\bar{\alpha}_{p, q, r} \in 7 \mathbb{Z}$ and $\alpha_{p, q, r}-\bar{\alpha}_{p, q, r} \in \sqrt{-7} \mathbb{Z}$. We complete the proof.

Remark 4.8. Using the character of $\operatorname{Aut}(C)=P S L_{2}\left(\mathbb{F}_{7}\right)$, we have $H^{0}\left(\operatorname{Aut}(C) ;\left(H_{\mathbb{C}}\right)^{\otimes_{\mathbb{C}} 3}\right)=$ $\mathbb{C}^{2}$. This induces $H^{0}\left(\operatorname{Aut}(C) ; H^{\otimes 3}\right)=\mathbb{Z}^{2}$. We can also prove that $\{(D+\bar{D}) / 7,(D-\bar{D}) / \sqrt{-7}\}$ is a generator of $H^{0}\left(\operatorname{Aut}(C) ;\left(H^{\otimes 3}\right)^{\prime}\right)$.

Theorem 4.9. The values at $(D+\bar{D}) / 7$ and $(D-\bar{D}) / \sqrt{-7} \in\left(H^{\otimes 3}\right)^{\prime}$ for the harmonic volume of the Klein quartic $C$ are given by

$$
0 \text { and } \frac{28}{\sqrt{-7}}\left(\frac{\zeta_{7}^{2}-\zeta_{7}^{6}}{\zeta_{7}+1} x_{1,2}+\frac{\zeta_{7}^{4}-\zeta_{7}^{5}}{\zeta_{7}^{2}+1} x_{2,3}+\frac{\zeta_{7}-\zeta_{7}^{3}}{\zeta_{7}^{4}+1} x_{3,1}\right) \bmod \mathbb{Z}
$$

respectively.

Proof. All iterated integral parts of $I((D+\bar{D}) / 7)$ and $I((D-\bar{D}) / \sqrt{-7})$ are linear combinations of $\int_{\ell_{k}} \omega_{i} \omega_{j}$ and $\int_{\ell_{k}} \bar{\omega}_{i} \bar{\omega}_{j}=\overline{\int_{\ell_{k}} \omega_{i} \omega_{j}}$. Furthermore, $\omega_{i} \wedge \omega_{j}=\bar{\omega}_{i} \wedge \bar{\omega}_{j}=0$. So we need no correction terms $\eta$ in Definition 2.1 Therefore, it suffices to calculate only the iterated integral parts.

By definition, there exist complex constants $\theta_{i, j, k}$ so that $I((D+\bar{D}) / 7)$ is of the form

$$
\sum_{k=1}^{7} \sum_{(i, j) \in U} \theta_{i, j, k} \int_{\ell_{k}}\left(\omega_{i} \omega_{j}-\omega_{j} \omega_{i}\right)+\sum_{k=1}^{7} \sum_{(i, j) \in U} \bar{\theta}_{i, j, k} \overline{\int_{\ell_{k}}\left(\omega_{i} \omega_{j}-\omega_{j} \omega_{i}\right)},
$$

where $U$ is a set $\{(1,2),(2,3),(3,1)\}$. Using P.D. $\left(\omega_{i}\right)=\lambda_{i} L_{7 h_{i}}=\lambda_{i} \sum_{k=1}^{7} \xi_{i}^{k} \ell_{k}$, it can be written as $\left(I_{1,2,3}+\bar{I}_{1,2,3}\right) / 7 \bmod \mathbb{Z}$. Here, we denote

$I_{1,2,3}=\lambda_{3} \sum_{k=1}^{7} \xi_{3}^{k} \int_{\ell_{k}}\left(\omega_{1} \omega_{2}-\omega_{2} \omega_{1}\right)+\lambda_{1} \sum_{k=1}^{7} \xi_{1}^{k} \int_{\ell_{k}}\left(\omega_{2} \omega_{3}-\omega_{3} \omega_{2}\right)+\lambda_{2} \sum_{k=1}^{7} \xi_{2}^{k} \int_{\ell_{k}}\left(\omega_{3} \omega_{1}-\omega_{1} \omega_{3}\right)$.

Similarly, we obtain

$$
I((D-\bar{D}) / \sqrt{-7})=\left(I_{1,2,3}-\bar{I}_{1,2,3}\right) / \sqrt{-7} \bmod \mathbb{Z} .
$$

In order to complete the proof, we need two lemmas. 
Lemma 4.10. We have

$$
\int_{\ell_{k}}\left(\omega_{i} \omega_{j}-\omega_{j} \omega_{i}\right)=2\left(\xi_{i} \xi_{j}\right)^{k-1}\left(1-\xi_{i} \xi_{j}\right) x_{i, j}+\left(\xi_{i} \xi_{j}\right)^{k-1}\left(\xi_{i}-1\right)\left(\xi_{j}+1\right) .
$$

Proof. We use Lemma 4.1. Lemma 4.5 and the equation

$$
\int_{\ell_{k}} \omega_{j} \omega_{i}=-\int_{\ell_{k}} \omega_{i} \omega_{j}+\int_{\ell_{k}} \omega_{i} \int_{\ell_{k}} \omega_{j}
$$

Lemma 4.11. We have

$$
I_{1,2,3}=14\left(\frac{\zeta_{7}^{2}-\zeta_{7}^{6}}{\zeta_{7}+1} x_{1,2}+\frac{\zeta_{7}^{4}-\zeta_{7}^{5}}{\zeta_{7}^{2}+1} x_{2,3}+\frac{\zeta_{7}-\zeta_{7}^{3}}{\zeta_{7}^{4}+1} x_{3,1}-\frac{3}{2} \sqrt{-7}\right)
$$

Proof. Using Lemma 4.10 and $\xi_{1} \xi_{2} \xi_{3}=1$, we calculate the coefficient of $x_{1,2}$ of $I_{1,2,3}$ as follows:

$$
\begin{aligned}
\lambda_{3} \sum_{k=1}^{7} \xi_{3}^{k} \cdot 2\left(\xi_{1} \xi_{2}\right)^{k-1}\left(1-\xi_{1} \xi_{2}\right) & =\frac{-2}{\xi_{3}^{3}\left(\xi_{3}^{2}+1\right)} \sum_{k=1}^{7}\left(\xi_{1} \xi_{2} \xi_{3}\right)^{k-1}\left(\xi_{3}-1\right) \\
& =\frac{-2}{\zeta_{7}^{12}\left(\zeta_{7}^{8}+1\right)} \sum_{k=1}^{7}\left(\zeta_{7}^{4}-1\right)=14 \frac{\zeta_{7}^{2}-\zeta_{7}^{6}}{\zeta_{7}+1} .
\end{aligned}
$$

Similarly, we compute the coefficients of $x_{2,3}, x_{3,1}$ and the constant term of $I_{1,2,3}$. For the computation of the constant term, we need $\zeta_{7}+\zeta_{7}^{2}+\zeta_{7}^{4}=(-1+\sqrt{-7}) / 2$.

The result follows from Lemma 4.11] We remark that all the coefficients of $x_{1,2}, x_{2,3}, x_{3,1}$ and the constant term of $I_{1,2,3}$ are pure imaginary.

For the numerical calculation of $x_{i, j}$, we recall the generalized hypergeometric function ${ }_{3} F_{2}$. We denote the gamma function $\Gamma(\tau)=\int_{0}^{\infty} e^{-t} t^{\tau-1} d t$ for $\tau>0$ and $(\alpha, n)=\Gamma(\alpha+n) / \Gamma(\alpha)$ for non-negative integer $n$. For $x \in\{z \in \mathbb{C} ;|z|<1\}$ and $\alpha_{1}, \alpha_{2}, \alpha_{3}, \beta_{1}, \beta_{2}>-1$, the generalized hypergeometric function ${ }_{3} F_{2}$ is defined by

$$
{ }_{3} F_{2}\left(\begin{array}{c}
\alpha_{1}, \alpha_{2}, \alpha_{3} \\
\beta_{1}, \beta_{2}
\end{array} ; x\right)=\sum_{n=0}^{\infty} \frac{\left(\alpha_{1}, n\right)\left(\alpha_{2}, n\right)\left(\alpha_{3}, n\right)}{\left(\beta_{1}, n\right)\left(\beta_{2}, n\right)(1, n)} x^{n} .
$$

See [8] for example. By straightforward computation, we have

Proposition 4.12. Let $\Delta$ be a 1-simplex $\left\{(u, v) \in \mathbb{R}^{2} ; 0 \leq v \leq 1,0 \leq u \leq v\right\}$. If $a, b, p, q>$ $0, b<1$, then we have

$\int_{\Delta} u^{a-1}(1-u)^{b-1} v^{p-1}(1-v)^{q-1} d u d v=\frac{B(a+p, q)}{a} \lim _{\substack{t \rightarrow 1-0 \\ t \in \mathbb{R}}} F_{2} F_{2}\left(\begin{array}{c}a, 1-b, a+p \\ 1+a, a+p+q\end{array} ; t\right)$.

From Proposition 4.12 we have

Lemma 4.13.

$$
x_{i, j}=\frac{B\left(h_{i}+h_{j}, h_{j+1}\right)}{h_{i} B_{i}^{\prime} B_{j}^{\prime}} \lim _{\substack{t \rightarrow 1-0 \\
t \in \mathbb{R}}} F_{2}\left(\begin{array}{c}
h_{i}, 1-h_{i+1}, h_{i}+h_{j} \\
1+h_{i}, h_{i}+h_{j}+h_{j+1}
\end{array} ; t\right) .
$$

Theorem 4.14. Let $C$ be the Klein quartic. Then, the cycle $C-C^{-}$is not algebraically equivalent to zero in $J(C)$. 
YUUKI TADOKORO

Proof. By Theorem 4.9 Lemma 4.13 the numerical calculation (Figure 1 in Appendix), we obtain the value

$$
2 I((D-\bar{D}) / \sqrt{-7})=0.72270 \pm 1 \times 10^{-5} \bmod \mathbb{Z} .
$$

The result follows from Proposition 3.2

\section{Appendix}

In this section, we introduce the MATHEMATICA program 14 in the proof of Theorem 4.14

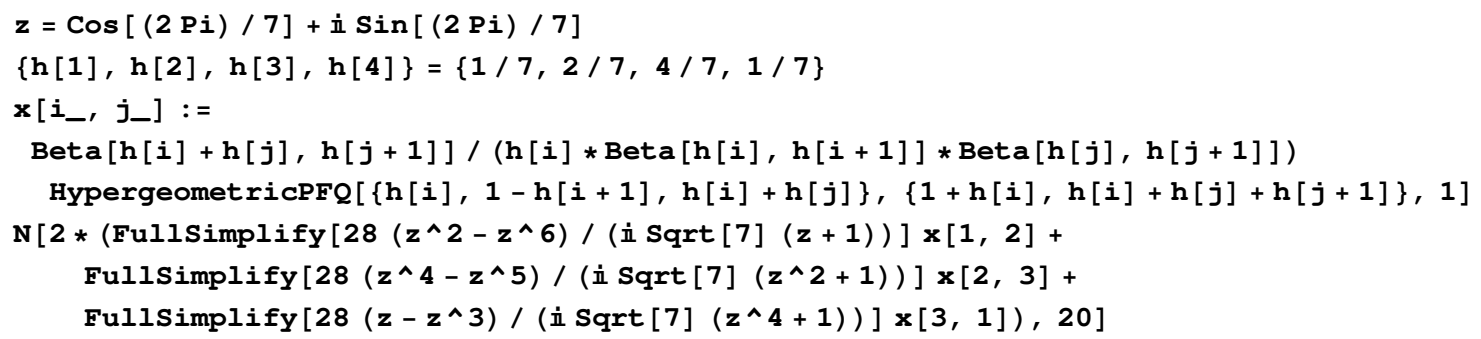

FiguRE 1. Numerical calculation program of Theorem 4.14

\section{REFERENCES}

[1] Ceresa, G.: $C$ is not algebraically equivalent to $C^{-}$in its Jacobian. Ann. of Math. (2) 117 (1983), no. 2, 285-291.

[2] Chen, Kuo Tsai: Algebras of iterated path integrals and fundamental groups. Trans. Amer. Math. Soc. 156 $1971359-379$.

[3] Hain, Richard M.: The geometry of the mixed Hodge structure on the fundamental group. Algebraic geometry, Bowdoin, 1985 (Brunswick, Maine, 1985), 247-282, Proc. Sympos. Pure Math., 46, Part 2, Amer. Math. Soc., Providence, RI, 1987.

[4] Harris, Bruno: Harmonic volumes. Acta Math. 150 (1983), no. 1-2, 91-123.

[5] Harris, Bruno: Homological versus algebraic equivalence in a Jacobian. Proc. Nat. Acad. Sci. U.S.A. 80 (1983), no. 4 i., 1157-1158.

[6] Harris, Bruno: Iterated integrals and cycles on algebraic manifolds. Nankai Tracts in Mathematics, 7. World Scientific Publishing Co., Inc., River Edge, NJ, 2004.

[7] Edited by Silvio Levy: The eightfold way. The beauty of Klein's quartic curve. Mathematical Sciences Research Institute Publications, 35. Cambridge University Press, Cambridge, 1999.

[8] Slater, Lucy Joan: Generalized hypergeometric functions. Cambridge University Press, Cambridge 1966.

[9] Pulte, Michael J.: The fundamental group of a Riemann surface: mixed Hodge structures and algebraic cycles. Duke Math. J. 57 (1988), no. 3, 721-760.

[10] Pirola, Gian Pietro: The infinitesimal invariant of $C^{+}-C^{-}$. Algebraic cycles and Hodge theory (Torino, 1993), 223-232, Lecture Notes in Math., 1594, Springer, Berlin, 1994.

[11] Tadokoro, Yuuki: The Harmonic Volumes of Hyperelliptic Curves, to appear in Publ. Res. Inst. Math. Sci.

[12] Tretkoff, C. L.; Tretkoff, M. D.: Combinatorial group theory, Riemann surfaces and differential equations. Contributions to group theory, 467-519, Contemp. Math., 33, Amer. Math. Soc., Providence, RI, 1984.

[13] Weil, Andre: Scientific works. Collected papers. Vol. II (1951-1964). Springer-Verlag, New YorkHeidelberg, 1979.

[14] Wolfram, Stephen: The Mathematica ${ }^{R}$ book. Fourth edition. Wolfram Media, Inc., Champaign, IL; Cambridge University Press, Cambridge, 1999.

Department of Mathematical Sciences, University of Tokyo, 3-8-1 Komaba, Meguro, Tokyo 153 8914, JAPAN

E-mail address: tado@ms.u-tokyo.ac.jp 